\title{
A Cardiac Surgery ICU Discharge Model; for Research and Logistic Purpose
}

\author{
Carl-Johan Jakobsen*, Alice Lundbøl Vestergaard, Erik Sloth, Anne Eldrup Vester and \\ Morten Nygaard
}

Department of Anaesthesia \& Intensive Care, Aarhus University Hospital, Skejby, Aarhus, Denmark

\begin{abstract}
Length of stay in the ICU is one of the factors limiting operating room utilization in cardiac surgery and consequently one of the primary cost-effectiveness parameters. At present it seems that factors guiding LOS in ICU after cardiac surgery has reached a stage, where the need for patient turnover, practical convenience and especially local policies are superior to what is actually possible and even many different recovery scores have been used and refined following new monitoring techniques length of stay is not a valid parameter when comparing institutions or procedures. ICU research and quality programmes are needed to make goal-oriented programmes. The purpose of this prospective study was to create an objective and reproducible ICU discharge model to be used in research and as an administrative logistic tool in cardiac surgery.

Patients and methods: All cardiac patients in a 50 day period $(\mathrm{N}=113)$ were followed. Our discharge model consists of 5 semi-objective variables (sedation, respiration, nausea, pain and motor function and 7 objective variables (peripheral saturation, diureses, arterial blood pressure, heart rate, cardiac index, temperature and postoperative drainage). Patients were score every hour until the next morning, termination of haemodynamic monitoring or at discharge, whichever came first. Patients were considered eligible for discharge from the cardiac recovery unit at a steady discharge score of 4 (IDS4) or below and with no single variable score higher than 2 .

Results: The actual discharge time was statistically significant longer than the eligible discharge time $(\mathrm{p}<0.0001)$, with a difference of 8.68 hours. The data showed no correlation between ventilation time and eligible time to discharge, while in actual discharge time patients ventilated shorter time also presented the shortest time to actual discharge.

Conclusion: Although very early extubation has impact on actual discharge time from ICU, it does not favour a shorter time to reach an eligible state. The perplexity of definitions in relation to LOS in ICU calls for an evaluation protocol and the described objective discharge model might be valuable, especially with respect to research, but also for logistic and administrative purposes. The relative objectivity makes comparison between units and centres more reliable than present reporting.
\end{abstract}

As a result of progressive cost containment in health care, providers of care are increasingly expected to produce reliable information regarding the cost-effectiveness of their procedures, which has resulted in a growing interest for fast extubation and early discharge from the intensive care unit (ICU) or Cardiac Recovery Unit (CRU). Length of stay (LOS) in the ICU is one of the factors limiting operating room utilization in cardiac surgery and consequently one of the primary cost-effectiveness parameters. Following, the main determinant of the augmented demand for "fast-track" cardiac surgery is the need for containing the burgeoning costs, saving resources and ICU time involved. Earlier studies have demonstrated that the application of fast-track cardiac anaesthesia results in a decreased postoperative ventilation time, resource usage and costs in patient's undergoing coronary artery bypass graft surgery [1]. Early extubation is classically considered one of the main steps in a fast-track process [2] and different anaesthesia protocols have been proposed to achieve this [3-8]. Regardless of the

*Address correspondence to this author at the Department of Anaesthesia \& Intensive Care, Aarhus University Hospital, Skejby, DK-8200 Aarhus N, Denmark; E-mail: cjj@dadlnet.dk anaesthetic regimen, there is still an open debate concerning optimal extubation time and time in ICU after cardiac surgery $[1,2,4,6,9]$.

For many years different recovery scores have been used [10-12] and refined following new monitoring techniques and methods [13-15]. The work with such programmes and the possibility to compare between institutions would benefit with agreed standards and definitions. However, focus during the last years has been ambulatory surgery $[16,17]$ and especially recovery after cardiac surgery has not been evaluated.

At present it seems that factors guiding LOS in ICU after cardiac surgery has reached a stage, where the need for patient turnover, practical convenience and especially local policies are superior to what is actually possible and thus ICU research and quality programmes are needed to make goal-oriented programmes with coordinated approach involving anaesthesia, surgery and nursing with constant reevaluation as events unfold, rather than rigid protocols. The purpose of this prospective study was to create an objective and reproducible ICU discharge model to be used in research and as an administrative logistic tool in cardiac surgery. 
Table 1. The Discharge Model

\begin{tabular}{|c|c|c|c|}
\hline \multicolumn{2}{|l|}{ Sedation } & \multicolumn{2}{|l|}{ Diureses (ml/kg/hour) } \\
\hline Awake & $\mathbf{0}$ & $>1$ & $\mathbf{0}$ \\
\hline Part time sleeping, awakening by verbal stimuli & 1 & $0.5-1.0$ & 1 \\
\hline Part time sleeping, awakening by physical stimuli & 2 & $0-0.5$ & 2 \\
\hline Sleeps, poor response stimulation & 3 & Anuria & 3 \\
\hline \multicolumn{2}{|l|}{ Respiration } & \multicolumn{2}{|l|}{ Blood pressure (mmHg) ${ }^{\text {b) }}$} \\
\hline Normal (Respiratory frequency $>10$ per minute) & $\mathbf{0}$ & $100-160$ & $\mathbf{0}$ \\
\hline Snoring (Respiratory frequency $>10$ per minute) & 1 & $90-99$ or $>160$ & 1 \\
\hline Respiratory frequency $<10$ & 2 & $81-89$ or $>170$ & 2 \\
\hline Apnoea periods or obstructive pattern per minute & 3 & $<80$ or $>180$ & 3 \\
\hline \multicolumn{2}{|l|}{ Pain (at rest) } & \multicolumn{2}{|l|}{ Heart rate (beats/minute) } \\
\hline None (VAS 0-1) & $\mathbf{0}$ & $50-100$ & $\mathbf{0}$ \\
\hline Light (VAS 2-4) & 1 & $101-120$ & 1 \\
\hline Moderate (VAS 5-7) & 2 & $<50$ or $>120$ or prophylactic pacemaker & 2 \\
\hline Severe (VAS 8-10) & 3 & $<40$ or $>130$ or pacemaker dependent & 3 \\
\hline \multicolumn{2}{|l|}{ Nausea } & \multicolumn{2}{|l|}{ Temperature $\left({ }^{\circ} \mathrm{C}\right)$} \\
\hline None & $\mathbf{0}$ & $\geq 36,0^{\circ}$ & $\mathbf{0}$ \\
\hline Light & 1 & $35,5-35,9^{\circ}$ & 1 \\
\hline Moderate & 2 & $35,0-35,4^{\circ}$ & 2 \\
\hline Severe (vomiting) & 3 & $<34.9^{\circ}$ & 3 \\
\hline \multicolumn{2}{|l|}{ Motoric function (in case of epidural) } & \multicolumn{2}{|l|}{ Cardiac Index $\left(\mathrm{L} / \mathrm{min} / \mathrm{m}^{2}\right)\left(\right.$ and SvO2) ${ }^{\mathrm{c})}$} \\
\hline Moves legs freely & $\mathbf{0}$ & $\mathrm{CI}>2.5$ & $\mathbf{0}$ \\
\hline Moves feet, bend knees & 1 & CI $2.2-2.5$ & 1 \\
\hline Only moves feet & 2 & CI $1.8-2.1$ & 2 \\
\hline No movement of legs & 3 & $\mathrm{CI}<1.8$ & 3 \\
\hline \multicolumn{2}{|l|}{$\mathrm{SpO}_{2(1-31 / \text { min oxygen supply })}{ }^{\text {a) }}$} & \multicolumn{2}{|l|}{ Drainage (ml/hour) } \\
\hline$\geq 94 \%$ & $\mathbf{0}$ & Drains removed & $\mathbf{0}$ \\
\hline $90-93 \%$ & 1 & $<20$ & 1 \\
\hline $85-89 \%$ & 2 & $21-50$ & 2 \\
\hline$<85 \%$ & 3 & $>50$ & 3 \\
\hline
\end{tabular}

the score is increased with 2 points; ${ }^{\circ}$ Continuous cardiac output measurements, if $\mathrm{SvO}_{2}$ is below $60 \%$ the score is increased with 2 points.

\section{PATIENT AND METHODS}

The study was approved by the local ethical committee as a quality control study. The study group comprised all patients scheduled for cardiac surgery procedures using cardiopulmonary bypass within a 50 days period. Patients who were not eligible for the specialized cardiac recovery unit (open from Monday till Saturday noon) for various reasons were a priori excluded from the study group.

\section{Discharge Model}

The variables and scores are shown in Table $\mathbf{1}$ and is a modified scoring system made for general surgery by Danish Society of Anaesthesia and Intensive Care ${ }^{1}$. The variables consist of 5 semi-objective variables (sedation, respiration,

\footnotetext{
${ }^{1}$ www.dasaim.dk/ (21-12-2008)
}

nausea, pain and motor function and 7 objective variables (peripheral saturation, diureses, arterial blood pressure, heart rate, cardiac index, temperature and postoperative drainage). Each variable was scored from 0 to 3 points by the nurses. Three variables could additionally be scored extra points; 1 point (saturation) or 2 points (blood pressure and cardiac index). Scoring started within 30 minutes after extubation and was continued every hour until the next morning until approximately 10 o'clock, termination of haemodynamic monitoring or at discharge, whichever came first.

Patients were considered eligible for discharge from the cardiac recovery unit at a steady discharge score of 4 (IDS4) or below and with no single variable score higher than 2 .

\section{Anesthesia and Haemodynamic Monitoring Protocols}

All preoperative cardiac medication was continued until the morning of surgery, with the exception of angiotensin- 
Table 2. Variables and Scores of the ICU/CRU Discharge Model. The Motoric Function Only Apply in Patients where Anaesthesia is Supplemented with Epidural Analgesia

\begin{tabular}{|l|c|c|c|c|c|c|c|c|c|}
\hline & \multirow{2}{*}{ No } & \multicolumn{2}{|c|}{ Ventilation Time (Hours) } & \multicolumn{2}{|c|}{ Time in ICU (Hours) } & \multicolumn{3}{|c|}{ Euro Score } & \multicolumn{2}{|}{ Age } \\
\cline { 3 - 10 } & & Mean & Range & Mean & Range & Mean & Range & Mean & Range \\
\hline \hline Study patients ${ }^{*}$ & 94 & 5.5 & $(1.7-16.9)$ & 21.4 & $(12.1-49.9)$ & 5.66 & $(0-13)$ & 64.8 & $(17-86)$ \\
\hline Expected prolonged ICU stay & 4 & 12.2 & $(2.5-22.0)$ & 312.9 & $(39.5-985.9)$ & 6.75 & $(2-11)$ & 69.3 & $(63-77)$ \\
\hline Special procedure & 7 & 40.0 & $(0.0-187.1)$ & 87.7 & $(22.1-384.4)$ & 6.57 & $(2-14)$ & 56.6 & $(43-68)$ \\
\hline No capacity in cardiac recovery $\left.{ }^{*}\right)$ & 4 & 6.0 & $(3.3-11.9)$ & 24.4 & $(21.9-27.9)$ & 6.00 & $(2-9)$ & 71.8 & $(69-75)$ \\
\hline Acute procedures & 4 & 5.8 & $(3.0-9.5)$ & 26.4 & $(15.8-42.3)$ & 11.50 & $(5-16)$ & 68.3 & $(61-75)$ \\
\hline All patients & 113 & 7.9 & $(0.0-187.1)$ & 36.1 & $(12.1-985.9)$ & 5.97 & $(0-16)$ & 64.8 & $(17-86)$ \\
\hline
\end{tabular}

All consecutive patients in the study period. ${ }^{*}$ ) No statistical significant differences between study patients and patients missed due to capacity in cardiac recovery.

converting enzyme inhibitors, aspirin and thrombocyte function inhibitors. All patients received standard benzodiazepine premedication 60-90 minutes before surgery. Anaesthesia consisted of total intravenous anaesthesia using Propofol 40-80 $\mu \mathrm{g} / \mathrm{kg} / \mathrm{min}$, Sufentanil 3-5 $\mu \mathrm{g} / \mathrm{kg}$ and Rocuronium $(0.8 \mathrm{mg} / \mathrm{kg})$. Standard monitoring included fivelead ECG, invasive radial arterial blood pressure, pulmonary artery catheter with continuous cardiac output measurement (Swan Ganz CCO/VIP; Edwards Lifesciences LLC, Irvine, CA), pulse oximetry, capnography, temperature monitoring and multiplane transoesophageal echocardiography.

\section{Surgical Procedure}

Routine surgical technique and cardio-protective strategies using crystalloid cardioplegia together with closed cardio-pulmonary bypass (CPB) systems were used in all patients. The CPB system consisted of tubing with a surface modifying additive coating, an arterial filter with heparin coating, a hollow fibre membrane oxygenator with a surfacemodified additive coating and a venous and cardiotomy reservoir. Most patients were kept normothermic or slightly hypothermic. Before weaning from CPB reperfusion of the heart was performed on an individual basis according to the patient's general condition and cross clamp time. There was no fixed postoperative treatment regimen for either pharmaceutical or mechanical support.

\section{Statistical Analysis}

The primary outcome variable was the eligible time of ICU discharge, expressed as the time when reaching a steady discharge score $\leq 4$ (IDS4). Further the eligible was compared to actual time of discharge. Perioperative variables were tested for association with early discharge using univariate analysis or logistic regression analysis. Comparisons between groups were tested using Student t-test for unpaired data, and categorical variables were tested using chi-square test or a relative risk analysis with $95 \%$ confidence intervals (CI) where appropriate. Data in tables are reported as mean, standard deviation of the mean or as count and percentage. A p $<0.05$ was considered significant for all the statistical tests. Statistic calculations were performed using the MedCalc ${ }^{\circledR}$ software version 9.5.1 (Mariakerke, Belgium).

\section{RESULTS}

113 consecutive patients underwent cardio-thoracic surgery procedures in the study period. Seven patients underwent special procedures like heart transplant and thoracic aortic surgery, 4 patients were for various reasons planned/expected to stay longer than 48 hours and another 4 patients underwent acute surgery during weekends and holidays. Those patients were excluded from final analysis together with 4 patients, who were not scored due to capacity problems in the cardiac recovery unit leaving 94 patients to be included in the study population (Table 2). Our study group was verified as a representative cohort of the cardiac surgery patients in 2007.

All patients, except one, reached the IDS4 without any single variable being higher than 2 . The referred patient had a score of 9 the morning after surgery and was discharged from the cardiac recovery after 49.9 hours. All other patients were discharged before 25.2 hours. Only one patient returned to ICU after 36 hours in ordinary ward.

Average and range of ventilation time and the time to reaching a score of 4 is shown in Table 3 . The actual discharge time was statistically significant longer than the time eligible discharge (a steady score of 4 or lower). Fig. (1) shows the number of patients reaching a steady score of $\leq$ 4 and no single score $\geq 3$ compared to actual discharge time.

Table 3. Ventilation Time, Eligible- and Actual Time to Discharge from Cardiac Recovery

\begin{tabular}{|l|c|}
\hline Average ventilation time & 5.54 \\
\hline Time to first IDS $\leq 4$ after extubation & 4.13 \\
\hline Time to steady IDS $\leq 4$ after extubation & 7.38 \\
\hline Total time to first IDS $\leq 4$ & 9.05 \\
\hline Total time to steady IDS $\leq 4$ & 12.29 \\
\hline Eligible length of stay $^{*}$ ) & 12.67 \\
\hline Actual length of stay in ICU $^{*}$ & 21.35 \\
\hline
\end{tabular}

Total times comprise of ventilation time plus time to IDS $\leq 4$. In six patients were observed a single observation variable of $\geq 3$ after patient has reached the IDS 4 , increasing the total time to eligible discharge 12.29 hours to 12.67 hours. $\left.{ }^{*}\right)$ The difference between eligible discharge (Total time to steady IDS $\leq 4$ no single $\geq 3$ ) and actual length of stay in ICU was statistical significant ( $p<0.0001$, Students t-test). IDS=ICU Discharge Score. Eligible length of stay $=$ total time to steady IDS $\leq 4$ and no single variable $\geq 3$. 


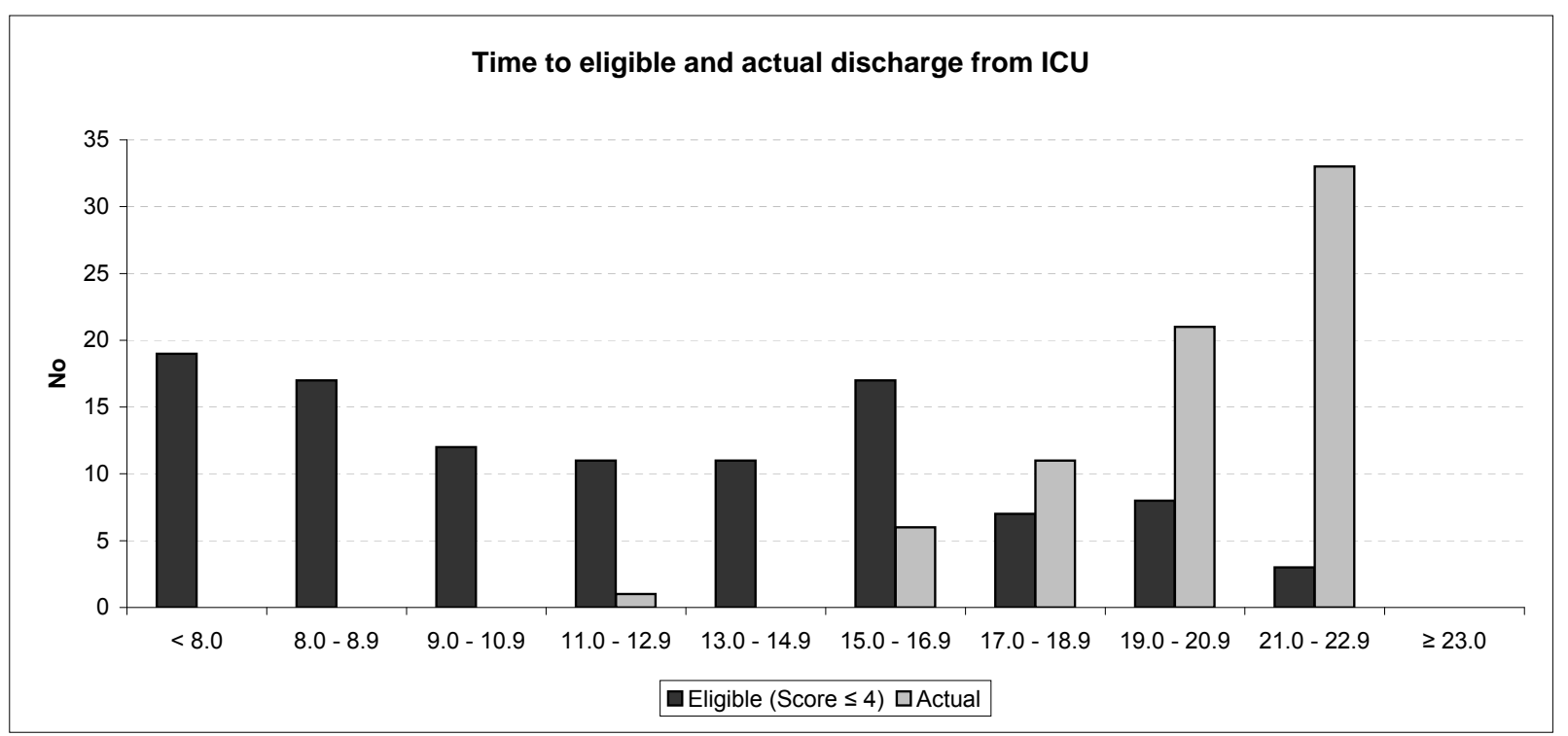

Fig. (1). Patients divided into time groups showing time to eligible discharge time (ISD4, steady score of $\leq 4$ and no single score $\geq 3$ ) and the actual discharge times. The difference between eligible and actual discharge score was statistical significant ( $p<0.0001$, chi-square test).

In six patients (6.4\%) the IDS4 was prolonged due to a single parameter $\geq 3$. This is shown in Table 4 which also demonstrates the total number and frequency of individual single scores $\geq 3$.

The impact of 7 factors on late IDS 4 and late actual discharge are shown in Table 5. Logistic regression analysis showed great differences between Odds-ratios in eligible and actual discharge, only female sex having the same oddsratio.

The data showed no correlation between time to IDS4 and ventilation time. Patients' extubated early had more events with early or later increase in IDS than patients' extubated late (Table 6).

Patients ventilated longer time reached IDS4 faster than patients extubated earlier and following there was no difference in eligible time to discharge (Table 7), while actual discharge was shorter in patients extubated early.

\section{DISCUSSION}

The analysis showed a statistical significant lower time

Table 4. Number of Individual Scores and Number of Individual Scores 3 or Higher, no of Patients with Score $\geq 3$ and the Number of Scores Prolonging the Time to Steady Score $\geq 4$

\begin{tabular}{|c|c|c|c|c|c|c|c|}
\hline \multirow{2}{*}{ Variable } & \multirow{2}{*}{$\begin{array}{c}\text { Scores } \\
\text { No }\end{array}$} & \multicolumn{2}{|c|}{ Events } & \multicolumn{2}{|c|}{ Patients } & \multicolumn{2}{|c|}{ Prolonged Score } \\
\hline & & No & $\%$ & No & $\%$ & No & $\%$ \\
\hline Total scores & 1270 & & & & & & \\
\hline Sedation & 1216 & 0 & $0,000 \%$ & & & & \\
\hline Respiration & 1241 & 1 & $0,081 \%$ & 1 & $1,064 \%$ & 0 & $0,000 \%$ \\
\hline Pain & 1100 & 15 & $1,364 \%$ & 10 & $10,638 \%$ & 2 & $2,128 \%$ \\
\hline Nausea & 1128 & 26 & $2,305 \%$ & 21 & $22,340 \%$ & 2 & $2,128 \%$ \\
\hline Motoric function & 1154 & 0 & $0,000 \%$ & & & & \\
\hline $\mathrm{SpO} 2$ & 1246 & 1 & $0,080 \%$ & 1 & $1,064 \%$ & 0 & $0,000 \%$ \\
\hline Diuresis & 1244 & 16 & $1,286 \%$ & 2 & $2,128 \%$ & 0 & $0,000 \%$ \\
\hline BP & 1246 & 19 & $1,525 \%$ & 8 & $8,511 \%$ & 0 & $0,000 \%$ \\
\hline HR & 1240 & 95 & $7,661 \%$ & 11 & $11,702 \%$ & 2 & $2,128 \%$ \\
\hline Temperature & 992 & 0 & $0,000 \%$ & & & & \\
\hline CI & 779 & 18 & $2,311 \%$ & 7 & $7,447 \%$ & 0 & $0,000 \%$ \\
\hline Drainage & 1139 & 73 & $6,409 \%$ & 31 & $32,979 \%$ & 0 & $0,000 \%$ \\
\hline Total & 13725 & 264 & $1,923 \%$ & 66 & $70,213 \%$ & 6 & $6,383 \%$ \\
\hline
\end{tabular}

Pain, Nausea, Diureses, Blood Pressure and Cardiac Index significant higher than Sedation, Respiration, Motoric function, SpO2 and Temperature and Drainage and Heart Rate significant different from all other. 
Table 5. Odd Ratio for Selected Variables on Late Eligible Discharge (Steady IDS $\leq 4$ ) and Actual Late Discharge

\begin{tabular}{|l|c|c|c|c|c|c|}
\hline \multirow{2}{*}{ Variable } & \multicolumn{3}{c|}{ Late Eligible Discharge } & \multicolumn{3}{c|}{ Actual Late Discharge } \\
\cline { 2 - 7 } & Odds Ratio & $\mathbf{9 5 \%}$ CI & P-Value & Odds Ratio & 95\% CI & P-Value \\
\hline \hline Age-score (EuroSCORE def) & 1.080 & $0.309-3.377$ & 0.904 & 1.948 & $0.640-5.933$ & 0.240 \\
\hline Female Sex & 1.496 & $1.233-16.160$ & 0.022 & 3.158 & $1.055-9.453$ & 0.039 \\
\hline S-Creatinine $>120 \mathrm{mmol} / 1$ & 14.371 & $2.073-99.661$ & 0.007 & 1.696 & $0.245-11.72$ & 0.593 \\
\hline Diabetes & 2.330 & $0.928-5.851$ & 0.072 & 1.003 & $0.409-2.457$ & 0.99 \\
\hline Valve surgery & 4.587 & $1.384-15.20$ & 0.001 & 1.232 & $0.387-3.923$ & 0.523 \\
\hline ECC time $\geq 120$ minutes & 0.399 & $0.088-1.796$ & 0.231 & 0.290 & $0.058-1.454$ & 0.132 \\
\hline Postoperative bleeding $\geq 1000 \mathrm{ml}$ & 12.60 & $2.635-60.32$ & 0.002 & 1.914 & $0.422-8.686$ & 0.400 \\
\hline
\end{tabular}

The only factors with impact on actual late discharge was female sex $(\mathrm{p}=0.039)$. Impact on late eligible discharge were female sex $(\mathrm{p}=0.022)$, preoperative $\mathrm{s}$-creatinine $>120 \mathrm{mmol} / 1$ $(\mathrm{p}=0.007)$, high postoperative bleeding $(\mathrm{p}=0.002)$ and valve surgery $(\mathrm{p}=0.001)$.

Definitions: Late eligible discharge is upper quartile of patients (eligible time $>15.9$ hours); Actual late discharge is upper quartile of patients (discharge $\geq 23.0$ hours); Age-score according to Euroscore definitions (from 1 (61 yr) to 6 (86 yr)); Diabetes=all types independent of type of treatment; Postoperative bleeding $\geq 1000 \mathrm{ml}$ during stay in ICU.

eligible discharge (IDS4 steady score $\leq 4$ and no single variable score $\geq 3$ ) than the time to actual discharge from ICU, being an average of 8.7 hours. The range of eligible discharge time was relatively great, being 2.9 hours to 22.4 hours.

In a recent study Ranucci at al [18] found that $21 \%$ of patients were discharged early from ICU (the morning after surgery). Not published data from a previously published study of more than 10.000 patients from 3 Danish institutions [19] showed that $80.3 \%$ was discharged within 24 hours after surgery and the data in this study showed that $82.3 \%$ was discharged from our ICU within 24 hours after surgery. In a preconditioning study, de Hert et al. [20] reported a differences in median LOS in ICU from 22 hours to 38 hours, depending on anaesthetic regime. In comparison our median LOS in ICU was 21.5 hours. Further de Hert et al. in another study [21] describe the normal ICU as 24 hours followed by 24 hours in a step down unit, while in our hospital only 6-8 \% is transferred to a step down unit and thus more than $72 \%$ of patients are transferred directly from our cardiac recovery to an ordinary ward within 24 hours. Basically there should not be such a big differences between major centres and this emphasize that LOS in ICU is dependent on factors other than strict medical indications, stressing the need for a well defined model for comparison.

The data in our study showed that time of extubation did have an impact on actual discharge from the ICU (Table 7). However adding ventilation time and time to reach IDS4 we saw no difference in eligible time of discharge (Table 7). Further we saw that patients extubated early had more episodes of set-back in IDS score (Table 6), which is in accordance with Ranucci [18] who revealed a significant

higher rate of post extubation complications in patients extubated after 2-3 hours versus patients extubated later.

Table 6. Number of Patients with Events Defined as an Increase in IDS of More Than 3 and Overall IDS Higher Than 6 After Initial Reach of IDS $\leq 4$, Correlated to Extubation Time

\begin{tabular}{|c|c|c|c|c|}
\hline Ventilation Time & Late $\uparrow$ & Early $\uparrow$ & None & All \\
\hline \hline Ventilation time $<4.0$ hours & 4 & 4 & 22 & 30 \\
\hline Ventilation time $4.0-5.9$ hours & 8 & 0 & 26 & 34 \\
\hline Ventilation time $>5.9$ hours & 1 & 1 & 28 & 30 \\
\hline All patients & 13 & 5 & 76 & 94 \\
\hline
\end{tabular}

The number of events was statistical significant more frequent in patients extubated before 5.9 hours ( $\mathrm{p}=0.024$, chi-square test).

Early increase is within 8 hours after extubation and late increase is more than 8 hours after extubation.

Only few studies have tried to evaluate on eligible and actual time of discharge from the ICU. Two studies used almost the same criteria; Patients were eligible to transfer out

Table 7. Influence of Ventilation Time on Mean Time to Reach IDS4, the Total Time (Ventilation Time + Time to Reach IDS 4) and the Actual Time of Discharge

\begin{tabular}{|c|c|c|c|}
\hline \multirow{2}{*}{ Ventilation Time } & \multicolumn{2}{|c|}{ Mean Hours to IDS4 } & \multirow{2}{*}{ Actual Time of Discharge (Mean Hours) $^{\text {\&) }}$} \\
\cline { 2 - 4 } & From Extubation $^{*}$ & Total $^{\text {!) }}$ & \\
\hline \hline Ventilation time $<4.0$ hours & 8,9 & 11.9 & 20.4 \\
\hline Ventilation time 4.0 -5.9 hours & 7,3 & 11.8 & 21.0 \\
\hline Ventilation time $>5.9$ hours & 5,9 & 14.4 & 22.7 \\
\hline All patients & 7,4 & 12.7 & 21.4 \\
\hline
\end{tabular}

*) The time to reach IDS 4 was shorter in patients ventilated longer $(\mathrm{p}=0.043)$. !) No difference was found in total time ( $\mathrm{p}=0.085)$. $)$ Patients ventilated shortest time had a statistical significant lower actual discharge time $(\mathrm{p}=0.043)$ (all Krushal-Vallis test). 
of the ICU when the following criteria were met: $\mathrm{SpO}_{2} \geq$ $90 \%$ at $\mathrm{FIO}_{2} \leq 0.5$ by facemask, adequate cardiac stability, no uncontrolled arrhythmia /no haemodynamically significant arrhythmia, chest tube drainage $<50 \mathrm{ml} / \mathrm{h}$, urine output $>0.5 \mathrm{ml} / \mathrm{kg} / \mathrm{h}$ and no intravenous inotropic or vasopressor therapy [21, 22]. Interesting, one of the studies show a difference less than 2 hours between eligible and actual time [21], while the study by Cheng et al. [22] showed a difference of 7-8 hours ( 0.3 days), the latter being in accordance with our findings of a difference of 8.7 hours.

The difference in factors having impact on late eligible and late actual time of discharge is interesting. That, postoperative bleeding, preoperative s-creatinine $>120$ $\mathrm{mmol} / \mathrm{l}$, valve surgery and perhaps female sex had negative impact on time to reach eligible discharge is expected. However, the findings that we could not demonstrate the same impact on actual late discharge, seems more surprising.

In conclusion it seems that although very early extubation has impact on actual discharge time from ICU, it does not favour a shorter time to reach an eligible state. The perplexity of definitions in relation to LOS in ICU calls for an evaluation protocol and the described objective discharge model might be valuable, especially with respect to research, but also for logistic and administrative purposes. The relative objectivity makes comparison between units and centres more reliable than present reporting.

\section{REFERENCES}

[1] Cheng DHC, Karski J, Peniston C, et al. Early tracheal extubation after coronary artery bypass graft surgery reduces costs and improves resource use: a prospective randomized controlled trial. Anesthesiology 1996; 85: 1300-10.

[2] Cheng DCH. Fast-track cardiac surgery pathways: early extubation, process of care, and cost containment. Anesthesiology 1998; 88: 1429-33.

[3] Westaby S, Pillai R, Parry A, et al. Does modern cardiac surgery require conventional intensive care? Eur J Cardiothorac Surg 1993; 7: 313-18.

[4] Cheng DHC, Karksi J, Peniston C, et al. Morbidity outcome in early versus conventional tracheal extubation following coronary artery bypass graft: a prospective randomized controlled trial. J Thorac Cardiovasc Surg 1996; 112: 755-64.

[5] Dowd NP, Karski JM, Cheng DC, et al. Fast-track cardiac anaesthesia in the elderly: effect of two different anaesthetic techniques on mental recovery. Br J Anaesth 2001; 86: 68-76.
[6] Engoren M, Luther G, Fenn-Buderer NC. A comparison of fentanyl, sufentanil, and remifentanil for fast-track cardiac anesthesia. Anesth Analg 2001; 93: 859-64.

[7] Tritapepe L, Voci P, Di Giovanni C, et al. Alfentanil and sufentanil in fast-track anesthesia for coronary artery bypass graft surgery. $\mathrm{J}$ Cardiothorac Vasc Anesth 2002; 16: 157-62.

[8] Brucek PJ, Straka Z, Vanek T, Jares M. Less invasive cardiac anesthesia: an ultra-fast-track procedure avoiding thoracic epidural analgesia. Heart Surg Forum 2003; 6: E107-10.

[9] Cheng DC, Wall C, Djaiani G, et al. Randomized assessment of resource use in fast-track cardiac surgery 1-year after hospital discharge. Anesthesiology 2003; 98: 651-7.

[10] Aldrete JA, Kroulik D. A postanesthetic recovery score. Anesth Analg 1970; 49: 924-34.

[11] Chung F. Are discharge criteria changing? J Clin Anesth 1993; 5: 64S-8S.

[12] Quinn CL, Weaver JM, Beck M. Evaluation of a clinical recovery score after general anesthesia. Anesth Prog 1993; 40: 67-71.

[13] Moller JT, Pedersen T, Rasmussen LS, et al. Randomized Evaluation of Pulse Oximetry in 20.802 Patients: II. Perioperative Events and Postoperative Complications. Anesthesiology 1993; 78: 445-53.

[14] Saar LM. Use of a modified Postanesthesia Recovery Score in phase II perianesthesia period of ambulatory surgery patients. J Perianesth Nurs 2001; 16: 82-9.

[15] Chung F. Discharge criteria-a new trend. Can J Anaesth 1995; 42: 1056-8.

[16] Chung F, Mezei G. Adverse outcomes in ambulatory anesthesia. Can J Anaesth 1999; 46: R18-R34.

[17] Marshall SI, Chung F. Discharge criteria and complications after ambulatory surgery. Anesth Analg 1999; 88: 508-17.

[18] Ranucci M, Bellucci C, Conti D, et al. Determinants of early discharge from the intensive care unit after cardiac operations. Ann Thorac Surg 2007; 83: 1089-95.

[19] Jakobsen C-J, Berg H, Hindsholm KB, et al. The influence in outcome of Propofol versus Sevoflurane anaesthesia in 10.535 single cardiac procedures. J Cardiothor Vasc Anesth 2007; 21: 66471 .

[20] De Hert SG, Van der Linden PJ, Cromheecke S, et al. Cardioprotective properties of sevoflurane in patients undergoing coronary surgery with cardiopulmonary bypass are related to the modalities of its administration. Anesthesiology 2004; 101: 299310.

[21] De Hert SG, Van der Linden PJ, Cromheecke S, et al. Choice of primary anesthetic regimen can influence intensive care unit length of stay after coronary surgery with cardiopulmonary bypass. Anesthesiology 2004; 101: 9-20.

[22] Cheng DC, Newman MF, Duke P, et al. The efficacy and resource utilization of remifentanil and fentanyl in fast-track coronary artery bypass graft surgery: a prospective randomized, double-blinded controlled, multi-center trial. Anesth Analg 2001; 92: 1094-102. 